\title{
Book report \\ Infectious diseases in the paediatric intensive care unit
}

Walter Zingg and Stephan Harbarth

\author{
Infection Control Program, University of Geneva Hospitals and Faculty of Medicine, 24 Rue Micheli-du-Crest, \\ $\mathrm{CH}-1211$ Geneva, Switzerland
}

Corresponding author: Stephan Harbarth, stephan.harbarth@hcuge.ch

Published: 25 July 2008

Critical Care 2008, 12:306 (doi:10.1186/cc6955)

This article is online at http://ccforum.com/content/12/4/306

(c) 2008 BioMed Central Ltd

Nadel S: Infectious Diseases in the Pediatric Intensive Care Unit. 1st edition. London: Springer; 2008, 580 pp. ISBN: 9781846289163

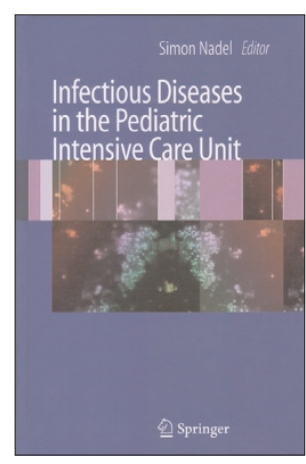

Infectious Diseases in the Pediatric Intensive Care Unit is a comprehensive collection of infectious disease topics in the paediatric intensive care unit (PICU) and in the neonatal intensive care unit (NICU) written by different experts. The chapters cover a broad range of interest, from the development of the immune system to diagnosis, treatment and prophylaxis of various infectious diseases including sepsis. The chapters on immunology, including the immunosuppressed host - with abundant information about congenital immunodeficiencies and HIV - are more comprehensive than other topics covered. There is further emphasis on potentially life-threatening tropical infections such as malaria, dengue, tetanus and Japanese encephalitis.

The purpose of the book is to provide a comprehensive overview of infectious diseases in critically ill neonates and infants. The scope of the book is broad, and it aims to provide information on pathophysiology as well as to serve as a practical manual on diagnosis, therapy and prevention of infectious diseases in the PICU and the NICU.

Infectious Diseases in the Pediatric Intensive Care Unit is primarily directed at physicians working with children in intensive care facilities who are not infectious diseases specialists. Most chapters of the book could also reach out to a larger audience, including medical students and infectious disease fellows who are not paediatricians. The short chapter on infection control might be of interest for nonspecialists; however, several of its paragraphs and references (for example, hand hygiene, epidemiology, antibiotic control) are outdated. Most chapters of the book are quite basic and therefore cannot replace currently available standard textbooks or state-of-the-art reviews.
Overall, this book does a good job of summarising and presenting the essentials of infectious diseases in the PICU and in the NICU in 580 pages. Moreover, it gives useful and practical information about diagnostic and therapeutic challenges. While some chapters are excellent, however, others are less so and - as the one about fungal infections are partially outdated and somewhat inaccurate due to recent publications in this field. Furthermore, the merged discussion of issues related to the NICU and the PICU makes some chapters difficult to read because there is considerable variation among differential diagnoses, therapies and prevention strategies among neonates versus older children. Finally, several chapters could have benefited from more illustrations and summary tables to facilitate quick reading.

This first edition of the Infectious Diseases in the Pediatric Intensive Care Unit provides comprehensive information about infectious diseases in paediatric and neonatal intensive care facilities. It is a useful guide for paediatric intensive care fellows and physicians in order to gain an overview of specific infectious disease topics in their daily practice.

\section{Conflicts of interest}

The authors declare that they have no competing interests.

$\mathrm{NICU}=$ neonatal intensive care unit; $\mathrm{PICU}=$ paediatric intensive care unit. 